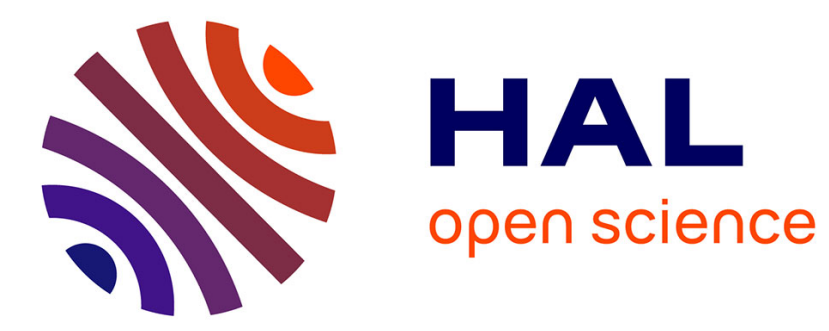

\title{
Les aphasies primaires progressives
}

M. Teichmann

\section{To cite this version:}

M. Teichmann. Les aphasies primaires progressives. Pratique Neurologique - FMC, 2019, 10, pp.96 100. 10.1016/j.praneu.2019.02.002 . hal-03486551

\section{HAL Id: hal-03486551 https://hal.science/hal-03486551}

Submitted on 20 Dec 2021

HAL is a multi-disciplinary open access archive for the deposit and dissemination of scientific research documents, whether they are published or not. The documents may come from teaching and research institutions in France or abroad, or from public or private research centers.
L'archive ouverte pluridisciplinaire HAL, est destinée au dépôt et à la diffusion de documents scientifiques de niveau recherche, publiés ou non, émanant des établissements d'enseignement et de recherche français ou étrangers, des laboratoires publics ou privés.

\section{(ㄷ)(1) $\$$}

Distributed under a Creative Commons Attribution - NonCommerciall 4.0 International 


\title{
Les aphasies primaires progressives
}

\section{Primary progressive aphasias}

\author{
Marc Teichmann ${ }^{1,2} *$ \\ ${ }^{1}$ Département de Neurologie, Institut de la Mémoire et de la Maladie d'Alzheimer, Centre de \\ Référence National 'Démences rares ou précoces'. Hôpital Pitié Salpêtrière, AP-HP, Paris, \\ France. \\ ${ }^{2}$ Institut du Cerveau et de la Moelle épinière (ICM), UMRS 975, ICM-INSERM 1127, \\ FrontLab team, Paris, France.
}

\section{*Adresse de correspondance}

Marc Teichmann

Département de Neurologie, Institut de la Mémoire et de la Maladie d'Alzheimer, Centre de Référence National 'Démences rares ou précoces'.

Hôpital de la Pitié Salpêtrière, 47-83, boulevard de l'Hôpital. 75013 Paris. France.

E-mail: marc.teichmann@psl.aphp.fr. Telephone: +331421675 34. Fax: +33142167504 


\title{
Résumé
}

Les aphasies primaires progressives (APP) représentent des maladies neurodégénératives qui affectent, aux stades débutants, sélectivement les différentes composantes du système anatomo-fonctionnel du langage. Les processus neuropathologiques sont variés et ne connaissent pas de corrélations robustes avec les phénotypes linguistiques et anatomiques.

L'article expose les variantes principales des APP en précisant leur expressions cliniques/langagières, leurs corrélats anatomiques et les différents mécanismes neuropathologiques sous-jacents. Il propose un guide pour le diagnostic et la démarche de classification des APP ainsi que des indications pour leur prise en charge et les perspectives thérapeutiques. Il rappelle aussi qu'en dehors des aspects médicaux et de recherche clinique, les APP représentent un modèle fondamental pour la recherche psycholinguistique et neurolinguistique visant à explorer davantage l'architecture fonctionnelle et l'implémentation cérébrale des processus du langage.

Key words: aphasies primaires progressives, caractérisation phénotypique, prise en charge, objectifs de recherche

\begin{abstract}
Primary Progressive Aphasias (PPA) are neurodegenerative diseases affecting selectively, at early stages, the different components of the anatomo-functional network dedicated to language processing. The causative neuropathological mechanisms are numerous and there is no robust correlation with linguistic and anatomical PPA phenotypes. The article describes the three main variants of PPA specifying their clinical/linguistic patterns, their anatomical underpinnings and the underlying neuropathological causes. It proposes a guide for PPA diagnosis and classification, as well as for patient follow-up and therapeutic issues. It is also a reminder that, beyond medical and clinical research issues, PPA represent a fundamental model for psycholinguistic and neurolinguistic research aiming at revealing progressively the functional architecture and cerebral implementation of language processes.
\end{abstract}

Mots-clés: Key words: primary progressive aphasias, phenotypical characterization, patient follow-up, research issues 


\section{Introduction}

L'aphasie primaire progressive (APP) correspond à un groupe syndromique de maladies neurodégénératives qui possèdent un tropisme particulier pour les structures cérébrales implémentant le langage. Par définition, l'atteinte langagière reste largement isolée pendant au moins deux ans, avant de s'étendre, dans la plupart des cas, à d'autres fonctions cognitives comme les processus exécutifs, la mémoire, les praxies ou le contrôle comportemental. L'APP connaît essentiellement trois formes phénotypiques qui se distinguent par leurs profils aphasiques et par la topographie lésionnelle. Elle touche des patients relativement jeunes, souvent avant 65 ans, entraînant ainsi un retentissement socioprofessionnel considérable. La prévalence peut être estimée à au moins 7/100 000 [1], ce qui correspond à environ 30004000 malades en France. Les APP sont des maladies invalidantes puisqu'elles affectent une capacité fondamentale de notre cognition, dont certains aspects comme la syntaxe et la structure du lexique sont propres à l'espèce humaine. L'atteinte du langage n'a pas d'impact majeur sur la capacité à générer des raisonnements mais elle entrave l'aptitude à les exprimer et/ou à les recevoir.

\section{Eléments d'histoire des APP}

Une des premières descriptions de patients ayant eu des troubles langagiers relativement isolés d'origine dégénérative remonte à Arnold Pick à la fin du 19ème siècle. La véritable histoire de recherche sur les APP commence cependant seulement en 1982 avec Marsel Mesulam [2], qui a décrit 5 patients caractérisés par une atteinte progressive et isolée des capacités linguistiques. Il a forgé le terme « aphasie primaire progressive » (primary progressive aphasia), qui est également connu sous la dénomination de syndrome de Mesulam. En 1989, Julie Snowden décrit un deuxième cadre syndromique, directement apparenté à l'APP, qu'elle appelle la « démence sémantique » (semantic dementia) [3]. Selon Julie Snowden il existerait un seul système sémantique de nature supra-modale dont l'atteinte entraîne non seulement un trouble de la sémantique verbale mais également des difficultés à accéder au sens de stimuli non-verbaux visuels, auditifs, olfactifs et tactiles. À l'inverse, Mesulam suggère l'existence d'une sémantique spécifiquement verbale pouvant être atteinte dans le cadre des APP. Cette controverse se reflète dans l'histoire des publications des critères diagnostiques, qui distinguent d'abord les APP de la démence sémantique [4] et qui proposent ensuite une forme d'APP caractérisée par des troubles sémantiques principalement dans la modalité verbale [5], pour parvenir au compromis actuel où les critères diagnostiques des 
APP qui stipulent qu'il existe une forme dite «APP variante sémantique », s'associant ou non à des troubles sémantiques multimodaux [6]. Bien que la question « d'un système sémantique versus plusieurs systèmes » soit toujours posée, la clinique factuelle nous enseigne qu'il existe trois variantes principales d'APP affectant : i) le sens des mots, ii) les formes des mots indépendamment du sens, et iii) l'agencement des mots et/ou des phonèmes et des traits phonétiques.

\section{Phénotypes langagiers des APP}

Les trois variantes principales sont représentées par l'APP non fluente/agrammatique, l'APP sémantique et l'APP logopénique. Les deux premières font partie des dégénérescences lobaires fronto-temporales alors que la variante logopénique dépasse ce cadre en impliquant des régions plus postérieures et supérieures empiétant sur le lobe pariétal. Chacune des trois variantes répond aux critères diagnostiques généraux d'APP, comprenant une atteinte progressive du langage qui reste isolée ou largement prédominante pendant au moins 2 ans et qui ne peut s'expliquer par un processus non dégénératif [6]. Les critères spécifiques actuels pour chacune des trois variantes d'APP ont été publiés par Gorno-Tempini et al. en 2011 [6]. Toutefois, il existe des patients atteints d'APP qui ne sont pas classables selon ces critères soit parce qu'ils présentent pas tous les signes requis pour diagnostiquer une variante particulière soit puisqu'ils présentent des signes de plusieurs variantes (APP mixtes) [7,8].

\section{APP nonfluente/agrammatique}

Elle est caractérisée linguistiquement par une atteinte syntaxique et de l'encodage phonologique (séquençage phonémique) à laquelle s'associe fréquemment une atteinte phonétique. Le discours est laborieux comprenant de nombreuses paraphasies phonémiques (substitutions et inversions de phonèmes et de syllabes) et des erreurs syntaxiques (p.ex., omission de mots de fonction ou de verbes, verbes non conjugués). Les productions peuvent également contenir des glissements phonétiques, définissant l'anarthrie, aussi appelée apraxie de la parole. L'occurrence isolée d'une apraxie de la parole caractérise un syndrome apparenté à l'APP nonfluente dite anarthrie primaire progressive (primary progressive apraxia of speech) [9]. La compréhension des patients atteints d'APP non fluente est globalement préservée sauf pour des phrases syntaxiquement complexes. De même, les capacités de dénomination sont grandement préservées [10]. L'évolution est caractérisée par une réduction progressive du langage parlé jusqu'à l'impossibilité complète d'émettre des mots. La communication reste possible par le canal écrit mais les troubles syntaxiques 
empêchent progressivement la formation de phrases et l'écriture aboutit à des mots juxtaposés, sans lien syntaxique. Parallèlement, les troubles s'étendent en dehors du langage en touchant principalement les fonctions exécutives et attentionnelles. Cette atteinte retentit progressivement sur l'axe de compréhension, ce qui mène à la diminution massive de toutes les performances langagières.

\section{APP sémantique}

Elle est caractérisée par une atteinte du système sémantique. Le discours est fluide mais «creux de sens ». Les patients ont des difficultés à produire des mots appropriés au contexte (manque du mot, paraphasies sémantiques) et à comprendre le sens des mots entendus et écrits. L'atteinte du sens évolue fréquemment vers d'autres modalités non-verbales, et notamment vers le traitement visuel, entraînant ainsi des troubles de la reconnaissance de visages et des difficultés à comprendre la signification d'objets. L'évolution est caractérisée par l'incapacité à accéder au sens des mots les plus courants et par une réduction progressive du discours qui, à terme, se limite à quelques formules de prédilection. En dehors de l'atteinte sémantique, on observe fréquemment l'apparition de troubles comportementaux, avec une attitude d'égocentrisme et des activités souvent « obsessionnelles » impliquant des domaines cognitifs préservés comme le calcul (p.ex., réalisation compulsive de sudokus). Dans un second temps émergent dans la plupart des cas des troubles dysexécutifs, qui contribuent à la perte progressive de l'autonomie.

\section{APP logopénique}

Elle se caractérise par une atteinte du lexique mentale et de la mémoire de travail verbale. Ceci se manifeste par un manque du mot qui est caractérisé par le phénomène dit du «mot sur le bout de la langue ». Les patients connaissent le sens du mot à produire mais ne parviennent pas à trouver sa forme sonore et écrite (atteinte lexicale), ce qui engendre de multiples pauses au sein du discours. L'axe de perception du langage est entravé par la diminution de la mémoire de travail verbale rendant difficile la compréhension et la répétition de phrases longues. L'APP logopénique évolue vers une dégradation de la compréhension de phrases excédant quelques mots et entraîne une réduction progressive du langage en raison du manque du mot. L'atteinte du lexique et de la mémoire de travail retentit plus généralement sur la qualité de la production langagière en entraînant de multiples pauses suivies de pertes du fil conducteur donnant ainsi lieu à un discours perçu comme décousu et diffluent. L'APP logopénique s'étend également au sein du système linguistique en entravant après quelques 
années d'évolution l'agencement des phonèmes (paraphasies phonémiques), les capacités sémantiques, et parfois la formation de structures syntaxiques. En dehors du langage, on observe habituellement une atteinte progressive des praxies idéomotrices, des capacités de calcul et de la mémoire épisodique.

\section{Atteintes cérébrales des APP}

Chacune des trois variantes d'APP est liée à des atteintes atrophiques de l'hémisphère dominant qui se distinguent par leurs localisations. La topographie des lésions a été explorée grâce à l'utilisation de la morphométrie corticale à partir de l'IRM. Selon la plupart des études, l'APP non fluente/agrammatique est liée à une plage d'atrophie intéressant le cortex frontal postéro-inférieur, englobant la région de Broca, et des portions antérieures de l'insula [11]. La variante sémantique est sous-tendue par une atteinte intéressant le pôle temporal à prédominance gauche, ainsi que des régions temporales inférieures et moyennes [11]. La forme logopénique est associée à l'atteinte du cortex temporal externe postéro-supérieur empiétant sur le cortex pariétal inférieur, en englobant «l'aire de Wernicke»[11]. L'ensemble de ces données a été complété par des études en imagerie nucléaire. Utilisant la scintigraphie cérébrale ou la tomographie par émission de positons au FluoroDeoxyGlucose (TEP-FDG), elles ont montré qu'il existe une concordance importante entre les zones d'atrophie et les régions ayant une perfusion défaillante et/ou une diminution du métabolisme cortical [12]. Enfin, les études en tractographie à partir de l'IRM de diffusion ont montré que les processus dégénératifs des APP ne se limitent pas au cortex mais qu'ils touchent également des faisceaux de connexion qui sont en lien avec les zones corticales endommagées [12].

\section{Neuropathologie des APP}

Les études neuropathologiques menées dans le cadre des APP ont montré l'implication essentiellement de deux types de lésions : celles habituellement associées aux dégénérescences lobaires fronto-temporales et celles rencontrées dans la maladie d'Alzheimer [13]. Dans le premier cas, il s'agit avant tout de lésions comportant des inclusions intraneuronales tau-positives ou ubiquitine-positives (TDP-43-positives), alors que dans le deuxième cas elles comportent la dégénérescence neurofibrillaire et des dépôts $\beta$-amyloïdes, s'organisant en plaques séniles. De manière générale, un tiers des APP semble lié à une

pathologie Alzheimer alors que les deux autres tiers sont en rapport avec des lésions tau- 
positives, ubiquitine-positives ou avec des lésions sans histologie distinctive. Bien qu'il n'existe pas de corrélation robuste entre des profils neuropathologiques et les différentes variantes d'APP, les données de la littérature indiquent que la variante nonfluente/agrammatique est majoritairement sous-tendue par des lésions tau-positives, la variante sémantique par des lésions ubiquitine-positives/TDP-43 et la variante logopénique par des lésions de type Alzheimer. Afin de détecter une pathologie Alzheimer in vivo, plusieurs auteurs ont utilisé des biomarqueurs du LCS (protéine tau et phospho-tau, peptide $\beta$ amyloïde) ou la TEP-amyloïde [14,15]. Les données de ces études sont globalement cohérentes avec les résultats neuropathologiques soulignant ainsi leur utilité dans la détermination in vivo de la nature du processus dégénératif chez un patient APP donné. Elles montrent également, comme la neuropathologie, que la proportion des lésions Alzheimer pour une variante d'APP donnée varie selon les études et qu'il n'existe pas de corrélation robuste entre les variantes cliniques et les processus neuropathologiques sous-jacents. De manière générale, la pathologie Alzheimer est retrouvée chez environ 5-30\% des APP sémantiques, 60-100\% des APP logopéniques, et 10-40\% des APP non fluentes/agrammatiques. Parmi les APP non fluentes/agrammatiques non-Alzheimer et tau-positives, certaines constituent l'expression initiale d'une dégénérescence cortico-basale (DCB) ou d'une paralysie supranucléaire progressive (PSP), et le tableau clinique s'enrichit souvent progressivement par des signes moteurs de parkinsonisme.

Concernant les formes ubiquitine-positives/TDP-43, il existe des formes d'APP génétiques de transmission autosomique dominante qui sont en rapport avec des mutations du gène de la progranuline $(P G R N)$. De même, des formes génétiques liées aux gènes C9ORF72, MAPT et TARDBP ont été décrites.

\section{Démarche diagnostique}

Le diagnostic des APP peut être difficile et leur classification demande une démarche systématique. Les patients se présentent fréquemment avec une plainte mnésique ou attentionnelle, et seul un interrogatoire précis du patient et de son entourage permet de relever que les troubles sont liés au dysfonctionnement du langage. Le diagnostic se fait ensuite en deux étapes qui consistent à vérifier les critères généraux définissant les APP (altération progressive du langage), et à classer le patient selon les critères spécifiques des trois variantes principales. Cette dernière étape peut s'avérer difficile quand l'APP est déjà évoluée, ce qui entraîne souvent une formule sémiologique d'APP mixte. En particulier l'APP logopénique connaît une vitesse d'évolution assez importante affectant fréquemment rapidement le 
système sémantique en plus du lexique et de la mémoire de travail verbale. Pour classer un patient donné il convient de déterminer au minimum : i) la fluence/fluidité du discours, ii) l'occurrence d'erreurs syntaxiques, de paraphasies sémantiques et phonémiques, et de glissements phonétiques, iii) les capacités de dénomination (lexique et sémantique), iv) les capacités de désignation d'objets/images sur proposition d'un mot, et de définition de mots (sémantique), v) la répétition de phrases (mémoire de travail verbale), et de mots phonologiquement complexes (encodage phonologique). Parmi ces items, les points ii), iv) et v) ont un pouvoir discriminatif important permettant de classer correctement plus de $95 \%$ des patients selon un algorithme simple [15] (Figure 1). Les patients qui ne sont pas classables par cet algorithme présentent souvent des formes très débutantes ou assez évoluées. Les formes évoluées s'exprimant fréquemment par un phénotype d'APP mixte doivent inciter à rechercher des lésions surajoutées, à envisager des diagnostics différentiels, et à rechercher des facteurs génétiques qui peuvent donner lieu à des phénotypes langagiers mixtes [16].

L'imagerie permet ensuite d'étayer le diagnostic. L'IRM encéphalique est indispensable pour rechercher des processus non dégénératifs dont la localisation dans les régions cérébrales dédiées incite parfois à écarter le diagnostic d'APP. Elle peut également fournir des arguments positifs pour l'APP sémantique en montrant une atrophie temporale antérieure de l'hémisphère dominant, qui est particulièrement bien visible sur des coupes coronales. L'IRM a peu de pouvoir discriminatif dans la variante non fluente/agrammatique et logopénique. La scintigraphie ou la TEP-FDG sont plus sensibles que l'IRM en permettant de montrer une hypoperfusion ou un hypométabolisme de la région fronto-rolandique/insulaire/péri-sylvienne (APP non fluente/agrammatique), de la jonction temporo-pariétale (APP logopénique) ou du pôle temporal (APP sémantique), de l'hémisphère dominant. Le dosage des biomarqueurs LCR ouvre ensuite une fenêtre sur la neuropathologie sous-jacente pouvant indiquer une pathologie Alzheimer.

Quant à la variante non fluente/agrammtique et l'anarthrie primaire progressive il convient de rechercher des signes moteurs qui orientent vers un cadre nosographique plus large de DCB ou de PSP qui peuvent se manifester initialement par des troubles aphasiques isolés. Devant des formes avec forte composante anarthrique il est également souhaitable de rechercher une atteinte de la corne antérieure par électropyographie.

\section{Prise en charge et suivi}

La prise en charge et le suivi des patients atteints d'APP n'est pas codifiée. Ils doivent tenir compte de l'évolution qui comporte habituellement une extension des troubles au sein du 
système linguistique et une atteinte progressive d'autres fonctions cognitives et du contrôle comportemental pouvant aboutir à une perte d'autonomie. La prise en charge et le diagnostic peuvent bénéficier de la contribution du Centre de Référence National « démences rares ou précoces » qui connaît un maillage de centres de compétence dont une des missions est la prise en charge des patients APP. De manière générale, un bilan de suivi orthophonique et neuropsychologique permet de conforter le diagnostic, d'extrapoler la vitesse d'évolution, et de fournir les cibles pour la rééducation orthophonique. Il n'y a pas lieu de répéter des explorations par imagerie si le diagnostic est posé et l'évolution est typique.

Quant aux mesures à visée thérapeutique, il n'existe actuellement aucun traitement médicamenteux validé. Les quelques essais pharmacologiques ayant utilisé entre autres des anti-cholinestérasiques ont porté sur de petites cohortes et/ou sur des patients sans caractérisation biomarqueurs LCS ou TEP-amyloide n'ont pas fourni de données concluantes. De même, il n'existe aucun consensus quant aux stratégies de la rééducation orthophonique qui dépend actuellement de l'évaluation subjective de l'orthophoniste. Il convient néanmoins d'initier une rééducation du langage, et des autres fonctions cognitives atteintes, qui est habituellement réalisée par les orthophonistes en ville. Les démarches médicosociales sont à envisager en fonction de l'évolution de la maladie.

L'absence de stratégies thérapeutiques dont la mise en place est conditionnée par une meilleure connaissance des APP a mené à une grande activité de recherche. Une des approches prometteuses est celle de la stimulation trans-crânienne ciblant spécifiquement des régions cérébrales implémentant les réseaux neuronaux du langage. Récemment une étude exploratoire dans l'APP sémantique en double aveugle contre placébo a démontré des effets modulatoires significatifs intra-sémantiques [17], ce qui ouvre la voie pour des essais à plus grande échelle visant à obtenir un effet thérapeutique par des mécanismes de neuroplasticité. Une autre approche possible est celle des rééducations linguistiques se basant sur une connaissance détaillée des processus affectés et préservés au sein du système du langage. L'approche pharmacologique, notamment dans les variantes avec positivité des biomarqueurs Alzheimer pourraient représenter une autre piste prometteuse.

\section{APP et recherche sur le langage}

En dehors de la recherche clinique les APP représentent un moteur pour la recherche plus fondamentale sur l'organisation des processus linguistiques et leur implémentation cérébrale. Ceci est possible grâce au modèle lésionnel que représentent les APP qui affectent, à des stades débutants, spécifiquement certaines composantes du système du traitement du langage 
et des structures cérébrales dédiées à ce traitement. Ainsi les APP ont par exemple permis de mieux comprendre l'architecture du lexique mental [18], l'organisation du système sémantique [19], et la découverte de l'épicentre des représentations/computations sémantiques qui est implémenté par les pôles temporaux [20]. Les APP ont l'avantage par rapport aux modèles vasculaires qu'elles comportent une atteinte plus homogène, du moins à des stades débutants, et qu'elles affectent l'ensemble des composantes anatomo-fonctionnelles du langage. En revanche, les modèles vasculaires sont alimentés par des cohortes nécessairement hétérogènes, et les localisations habituelles des AVC ne comprennent pas toutes les régions du langage, épargnant par exemple les pôles temporaux. Ainsi, les APP sont devenu un 'outil' précieux pour répondre à des questions à la fois psycholinguistiques, neurolinguistiques et, de manière plus générale, aux interrogations des neurosciences cognitives.

\section{Liens d'intérêts}

Les auteurs n'ont pas transmis leurs liens d'intérêts éventuels en relation avec cet article. 


\section{Points essentiels}

- Les aphasies primaires progressives comprennent trois variantes auxquels s'ajoutent des formes 'non-classables' qui reflètent fréquemment des formes débutantes ou évoluées.

- Les liens entre atteinte cérébrale et phénotype langagier sont robustes mais les causes neuropathologiques ne connaissent que des corrélations tendancielles avec les différentes variantes d'APP.

- La démarche diagnostique des trois variantes d'APP suit des démarches systématiques et élaborées par plusieurs auteurs.

- Il n'y pas de thérapie validée pour les APP mais il existe plusieurs pistes prometteuses.

- En dehors de l'aspect clinique, les APP représentent un modèle unique pour l'exploration les substrats anatomo-fonctionnels du langage. 


\section{Références}

[1] Ratnavalli E, Brayne C, Dawson K, Hodges JR. The prevalence of frontotemporal dementia. Neurology 2002; $58: 1615-21$.

[2] Mesulam M. Slowly progressive aphasia without generalized dementia. Ann Neurol 1982; $11: 592-8$.

[3] Snowden JS, Goulding PJ, Neary D. Semantic dementia : a form of circumscribed cerebral atrophy. Behav Neurol 1989; 2 : 167-82.

[4] Neary D, Snowden JS, Gustafson L, Passant U, Stuss D, Black S, et al. Frontotemporal lobar degeneration : a consensus on clinical diagnostic criteria. Neurology 1998; 51 : 1546-54.

[5] Mesulam M. Primary progressive aphasia. Ann Neurol 2001; 49 : 425-32.

[6] Gorno-Tempini ML, Hillis AE, Weintraub S, Kertesz A, Mendez M, Cappa SF, et al. Classification of primary progressive aphasia and its variants. Neurology 2011; 76 : 1006-14.

[7] Harris JM, Gall C, Thompson JC, Richardson AM, Neary D, du Plessis D, et al. Classification and pathology of primary progressive aphasia. Neurology 2013; 81:1832-9.

[8] Mesulam MM, Weintraub S. Is it time to revisit the classification guidelines for primary progressive aphasia? Neurology 2014; 82: 1108-9.

[9] Josephs KA, Duffy JR, Strand EA, Machulda MM, Senjem ML, Master AV, et al. Characterizing a neurodegenerative syndrome: primary progressive apraxia of speech. Brain 2012;135: 1522-36.

[10] Migliaccio R, Boutet C, Valabregue R, Ferrieux S, Nogues M, Lehéricy S, et al. The Brain Network of Naming: A Lesson from Primary Progressive Aphasia. PLoS One 2016; 11(2):e0148707

[11] Gorno-Tempini ML, Dronkers NF, Rankin KP, Ogar JM, Phengrasamy L, Rosen HJ, et al. Cognition and anatomy in three variants of primary progressive aphasia. Ann Neurol 2004; $55: 335-46$.

[12] Routier A, Habert MO, Bertrand A, Kas A, Sundqvist M, Mertz J, et al. Structural, Microstructural, and Metabolic Alterations in Primary Progressive Aphasia Variants. Front Neurol 2018; 9:766. doi: 10.3389/fneur.2018.00766.

[13] Mesulam MM, Weintraub S, Rogalski EJ, Wieneke C, Geula C, Bigio EH. Asymmetry and heterogeneity of Alzheimer's and frontotemporal pathology in primary progressive aphasia. Brain 2014 ; 137:1176-92. 
[14] Hu WT, McMillan C, Libon D, Leight S, Forman M, Lee VM, et al. Multimodal predictors for Alzheimer disease in nonfluent primary progressive aphasia. Neurology 2010; $75: 595-602$.

[15] Leyton CE, Villemagne VL, Savage S, Pike KE, Ballard KJ, Piguet O, et al. Subtypes of progressive aphasia : application of the international consensus criteria and validation using $\beta$ amyloid imaging. Brain 2011; 134 : 3030-43.

[16] Rohrer JD, Crutch SJ, Warrington EK, Warren JD. Progranulin-associated primary progressive aphasia: a distinct phenotype? Neuropsychologia 2010; 48: 288-297.

[17] Teichmann M, Lesoil C, Godard J, Vernet M, Bertrand A, Levy R, et al. Direct current stimulation over the anterior temporal areas boosts semantic processing in primary progressive aphasia. Ann Neurol 2016; 80:693-707.

[18] Sanches C, Routier A, Colliot O, Teichmann M. The structure of the mental lexicon: What primary progressive aphasias reveal. Neuropsychologia 2018; 109: 107-115.

[19] Mesulam MM, Wieneke C, Hurley R, Rademaker A, Thompson CK, Weintraub S, et al. Words and objects at the tip of the left temporal lobe in primary progressive aphasia. Brain. 2013;136:601-18.

[20] Patterson K, Nestor PJ, Rogers TT. Where do you know what you know? The representation of semantic knowledge in the human brain. Nat Rev Neurosci. 2007;8:976-87. 


\section{Légende}

Figure 1. Algorithme de classification des différentes variantes d'Aphasies Primaires Progressives (APP). Adapté de Leyton et al. [15]. 


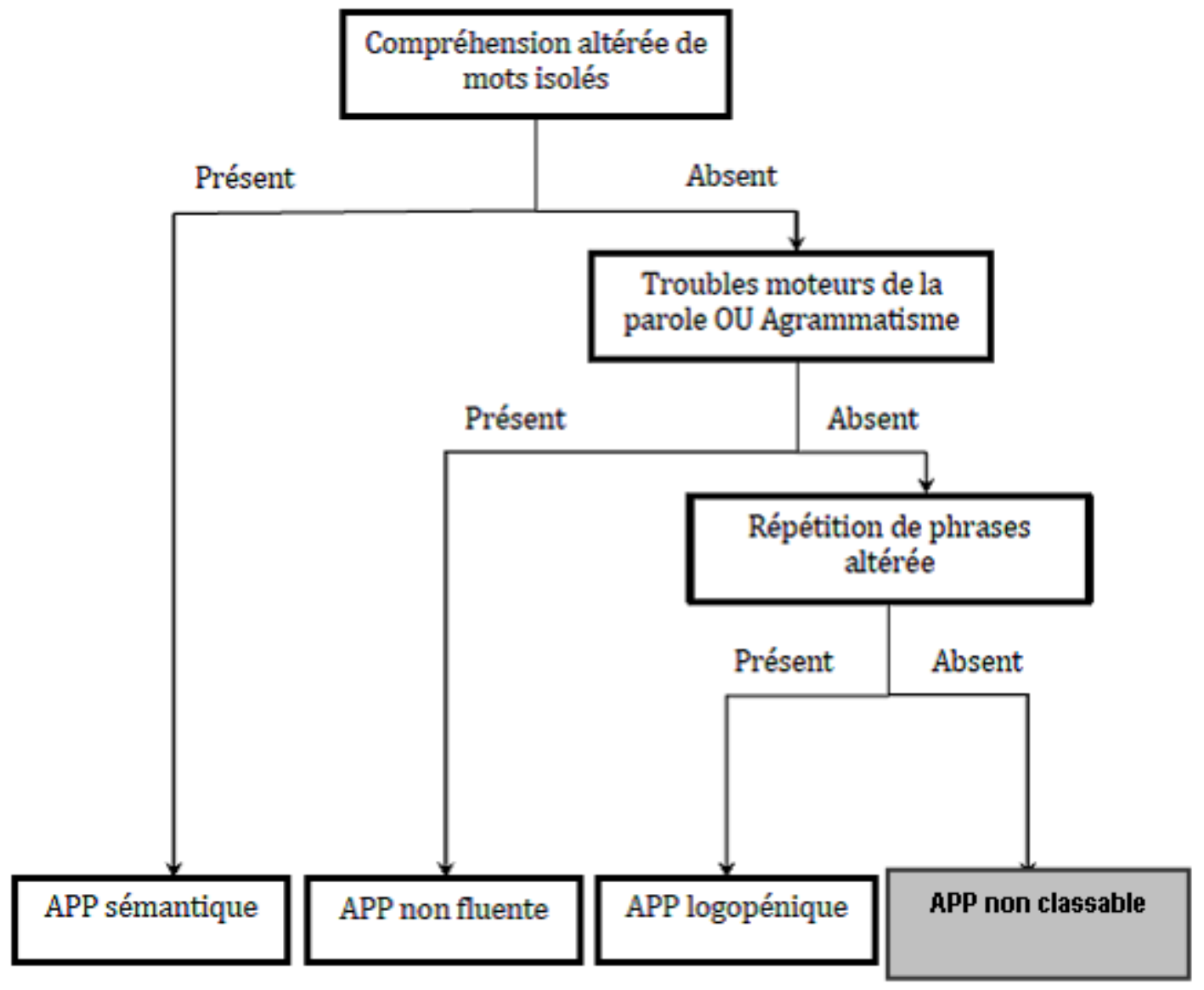

